\title{
DTM GENERATION IN FOREST REGIONS FROM SATELLITE STEREO IMAGERY
}

\author{
J. Tian, T. Krauss, P. Reinartz \\ Remote Sensing Technology Institute, German Aerospace Center (DLR), 82234 Wessling, Germany \\ (Jiaojiao.Tian, Thomas.Krauss, Peter.Reinartz)@dlr.de
}

Commission I, WG I/4

KEY WORDS: Optical Stereo Data, DTM, DSM, Forest, Random Forests

\begin{abstract}
:
Satellite stereo imagery is becoming a popular data source for derivation of height information. Many new Digital Surface Model (DSM) generation and evaluation methods have been proposed based on these data. A novel Digital Terrain Model (DTM) extraction method based on the DSM from satellite stereo imagery is proposed in this paper. Instead of directly filtering the DSM, firstly a single channel based classification method is proposed. In this step, no multi-spectral information is used, because for some stereo sensors, like Cartosat-1, only panchromatic channels are available. The proposed classification method adopts the random forests method to get initial probability maps of the four main classes in forest regions (high-forest, low-forest, ground, and buildings). To cover the pepper and salt effect of this pixel based classification method, the probability maps are further filtered based on the adaptive Wiener filtering. Then a cube-based greedy strategy is applied in generating the final classification map from these refined probability maps. Secondly, the height distances between neighboring regions are calculated along the boundary regions. These height distances can be used to estimate the relative region heights. Thirdly, the DTM is extracted by subtracting these relative region heights from the DSM in the order of: buildings - low forest - high forest. In the end, the extracted DTM is further smoothed using median filter.
\end{abstract}

The proposed DTM extraction method is finally tested on satellite stereo imagery captured by Cartosat-1. Quality evaluation is performed by comparing the extracted DTMs to a reference DTM, which is generated from the last return airborne laser scanning point cloud.

\section{INTRODUCTION}

Digital Terrain Models (DTMs) provide essential information for many remote sensing projects and applications. A DTM can be extracted from a Digital Surface Model (DSM) automatically by removing nonground objects and filtering these areas with proper elevation values (Kraus and Pfeifer, 2001; Arefi et al., 2011; Krauss et al., 2011). When both models DSM and DTM are available, the absolute height of buildings and trees can be calculated.

Various DTM generation methods have been proposed when different sources of DSMs are used. Photogrammetry and laser scanning are still two of the most important approaches in DSM/DTM generation. A detailed description and comparison between photogrammetry and airborne laser scanning (ALS) approaches can be found in Baltsavias (1999). Generally, ALS data are more advanced for DTM generation. In urban regions, ALS data are more accurate and building boundaries are modeled better. In forest regions, most of the ALS last return points show already the ground pixels, and trees are well separated from each other. A DTM can be generated by filtering these remaining trees.
Compared to ALS data, the DSMs generated from satellite stereo imagery (stereo-DSM) are more economical data sources and also advantageous for larger region survey and mapping applications (Zhang et al., 2005; d'Angelo et al., 2010; Tian et al., 2013). Moreover, the resolution as well as the quality of the extracted DSMs is improving (Straub et al., 2013). However, not many specific DTM extracting methods have been proposed for these data. Directly filtering the DSMs (Pfeifer et al., 2001; Arefi et al., 2011) will not work so well in dense forest regions, since in the stereo-DSMs, ground height between trees cannot be observed.

In this paper, a novel DTM generation method is proposed specifically for stereo-DSM in forest regions. Instead of directly filtering the DSM, the original satellite image is used to provide an initial classification result. To cover the pepper and salt effect of this pixel based classification method, a refined random forest classification method is introduced. Besides classification, in the second section, the step down DTM interpretation workflow is described. The experiments and results are shown and evaluated in the third section. The last section is conclusion. 


\section{METHODS}

A three steps DTM generation procedure is proposed in this paper. As shown in Fig. 1, in the first step, the orthorectifed satellite panchromatic images are used to get an initial classification result. Based on the classification results, the terrain height can be calculated from lower objects to higher objects. In the end, a further refinement of the DTM is applied.

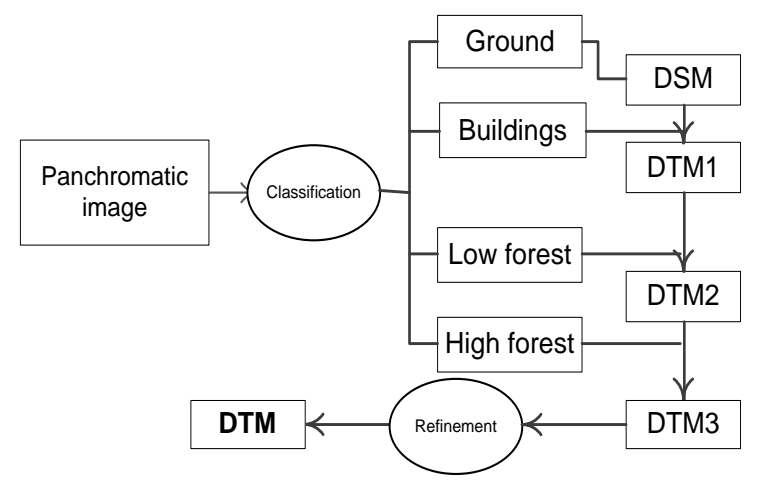

Figure 1. Flow chart of the proposed method

\subsection{Classification}

Random Forest is a robust and powerful machine learning classifier, which is capable of processing large datasets (Breiman, 2001). The random forest consists of several decision trees, also called classification trees. These classification trees are "grown" based on training sets. Random forest builds the tree nodes randomly with random features. Each decision tree is used separately to classify the satellite image of interest. By summarizing these classification results, one classification map and several membership maps for each class can be obtained.

In this step, no multi-spectral information is used, because for some stereo sensors, like Cartosat-1, only panchromatic channels are available. The proposed classification method adopts the multi-level features explained in Tian et. al (2013) to get initial probability maps of the four main classes in forest regions. These four classes are high-forest, low-forest, ground, and buildings.

To overcome the drawback of the pixel based classification method, which leads to a salt-and-pepper effect, two approaches are considered in this step. Besides the classification result, random forest can also provide the probability map of all classes. Therefore, an adaptive Wiener filtering is applied to improve the quality of each probability map. Afterwards, instead of only considering the probability sets pixel wise, their neighborhood pixels are also considered in the decision making procedure. It can be called 'cube-based greedy strategy'. The class label which has the highest probability in this probability cube is selected as the label of the pixel.

\subsection{Step down DTM generation}

Different to urban regions, in forest regions large areas of higher objects are connected together, which makes the DTM generation in forest regions more difficult. In this paper, we tried to separate large forest regions into a number of small regions by using the classification result. The relative heights of three high-level object classes are calculated respectively.

As shown in Figure 2, in each step, the height distance between the higher objects and lower objects can be calculated. The lower forest and higher forest canopy are shown in green and light blue color respectively. The red point represents one obtained boundary pixels. The yellow rectangle is the pre-defined search window. We take the height distance inside this search window as the step height of that red pixel. For each high object region, the object height is obtained by calculating the average step height $\Delta H$ of all boundary pixel, which means the height distance of $h_{1}$ and $h_{2}$.

The DTM is extracted by subtracting these relative region heights from the DSM in the order of: buildings - low forest - high forest. The building class is processed as the first step, as it is mainly separated from the forest region. Figure 2 focuses on explaining the DTM generation for low / high forest areas.

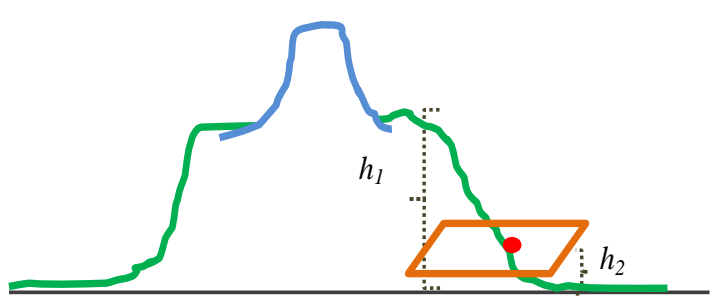

(a) (Step1)

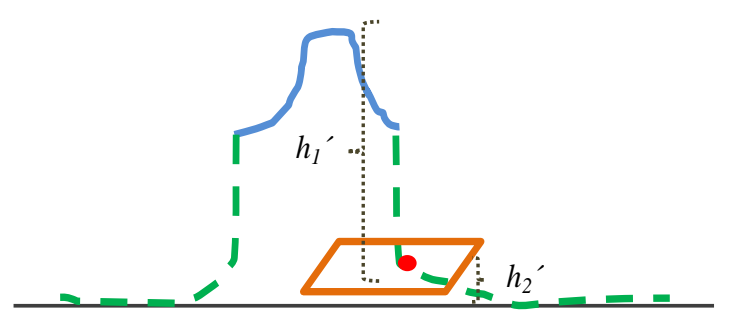

(b)

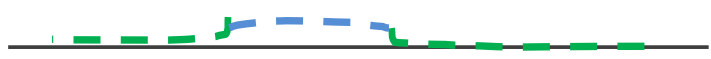

(c)

Figure 2. Step down height calculation method (green: low forest, blue: high forest; black: ground surface) 


\subsection{DTM refinement}

A further refinement of the generated DTM is necessary. Since the regions in each class have been processed separately, there would be a height difference remaining in the boundary region. Therefore a median filtering is adopted to improve the quality of the generated DSM.

\section{EXPERIMENT AND RESULTS}

\subsection{Datasets}

In this paper, Cartosat-1 stereo imagery captured in Traunstein, Germany are selected for the experiment. Figure $3 \mathrm{a}$ is the panchromatic image. The generated DSMs from Cartosat-1 are displayed in Figure $3 \mathrm{~b}$ and 3c respectively. The DSM generation procedure and quality evaluation have been described in Tian et al. (2013) and Straub et al. (2013).

To validate the accuracy and efficiency of the proposed method the DTM from Laser scanning data is used as reference data (shown in Figure 3c). All Cartosat-1 images have been resampled to 2.5 meter resolution.

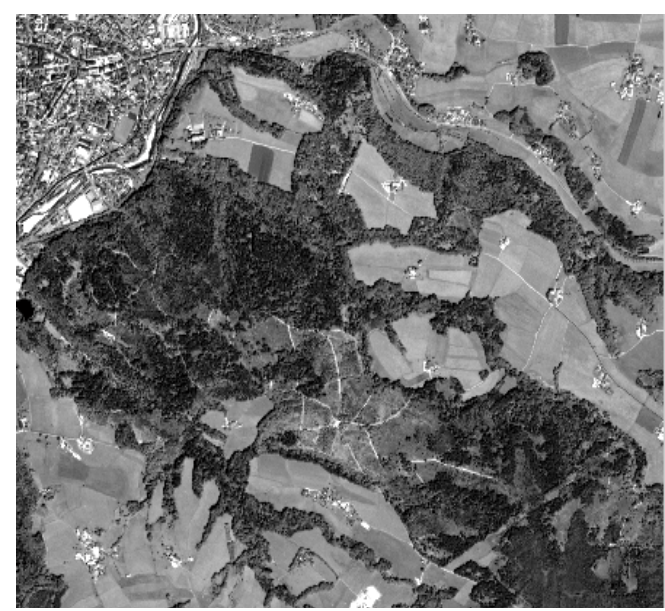

(a)

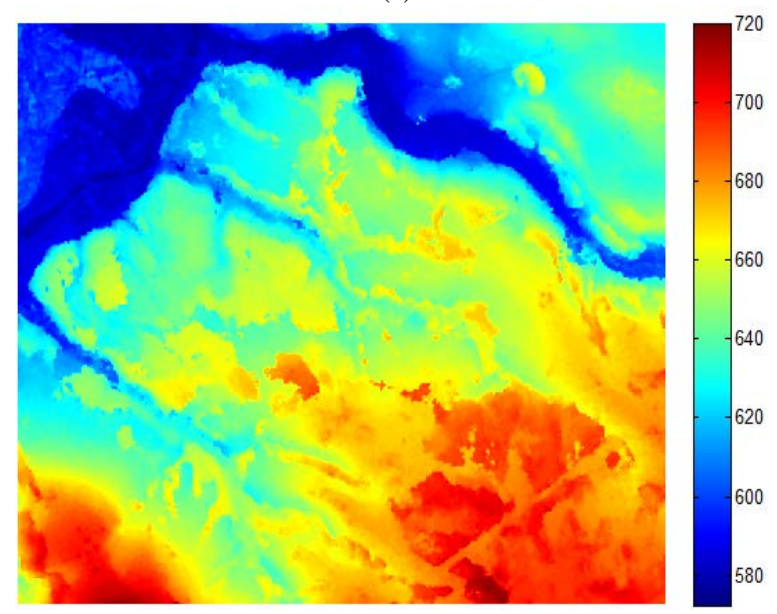

(b)

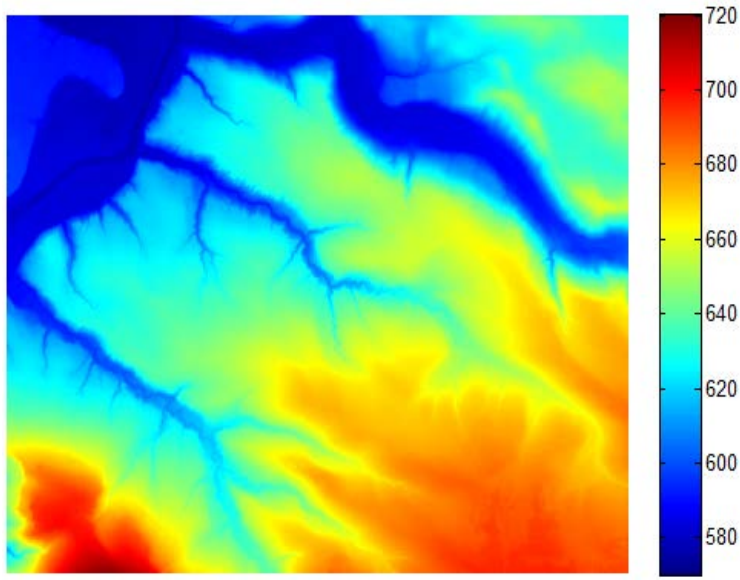

(d)

Figure 3. Experimental datasets: (a) panchromatic image; (b) DSM from Cartosat-1; (c) reference DTM

\subsection{Results}

\subsubsection{Classification}

The classification result for Carosat-1 is shown in Figure $4 \mathrm{~b}$. The tour classes are marked with red, light green, dark green and grey respectively. In order to show the improvement, the original classification result from random forests is displayed in Figure 4a. By referring to Figure 3a, the original random forest can label these four classes correctly. Limited to the salt-and-pepper effect, DTM generation cannot be easily performed. The proposed classification result (shown in Figure $4 \mathrm{~b}$ ) is visually much better. Different classes are well separately from each other. It can be observed that high forest regions are mainly surrounded by lower forest. Though some roads are wrongly classified as buildings, we will process them in the first step. Since they do not have height distance to the ground, they will keep their original height in DTM1.

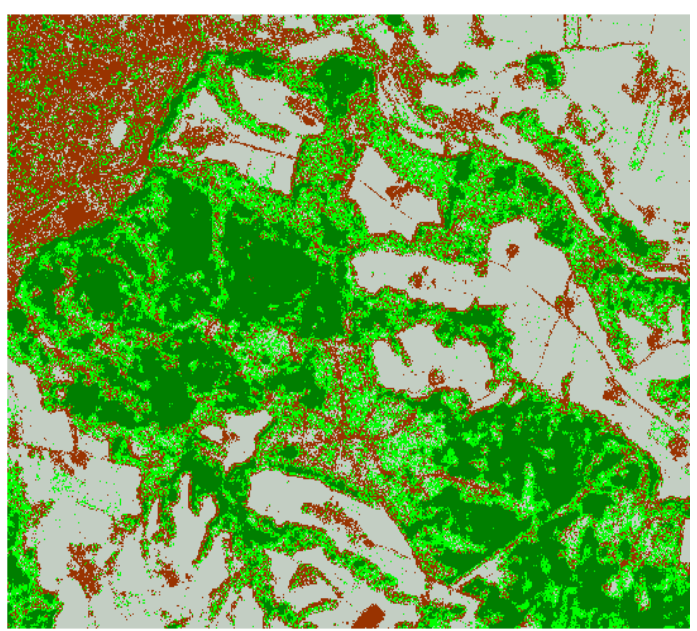

(a) 


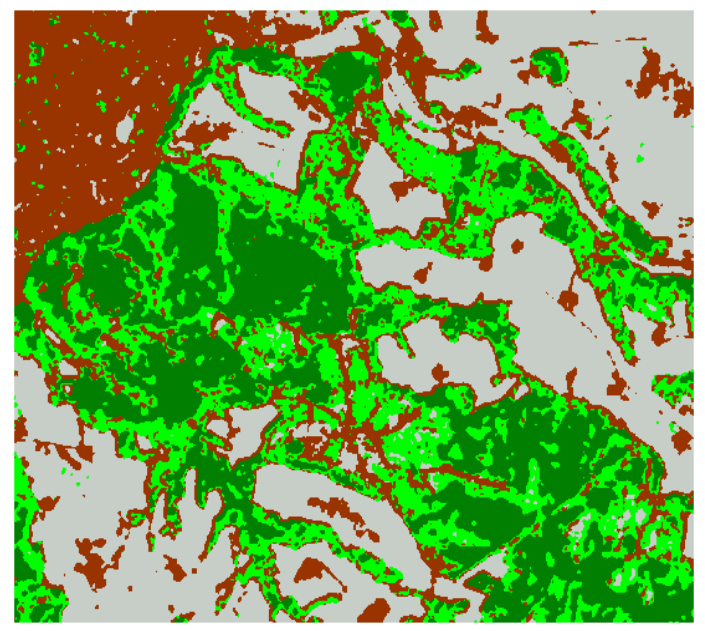

(b)

Figure 4. Comparison of the original classification map (a) and the refined classification results (b). (Red: buildings; Grey: ground; Dark green: high forest; Light green: low forest).

\subsubsection{DTM generation}

In the second part we evaluate the behavior of the DTM generation method. Two regions in the test data are analyzed, one is a forest region with high/low forest, and the other has a house in the middle of it. The results from Cartosat- 1 in each step are recorded in Figure 5 and Figure 6 respectively. For the forest region, we have displayed here all three DTMs in the generation procedure and the final refined DTM (Figure 5f). Figure $5 \mathrm{~g}$ is the reference DTM. In Figure 6, since no forest exists around that house, only DTM1 is generated.

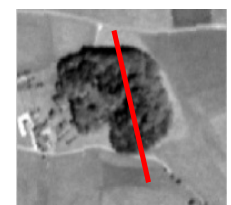

(a)

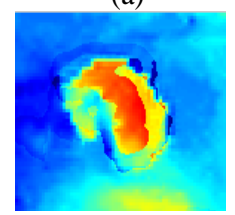

(d)

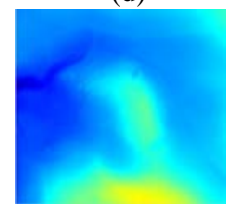

(g)

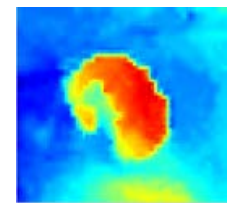

(b)

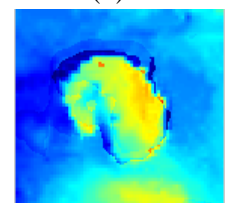

(e)

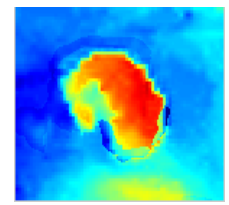

(c)

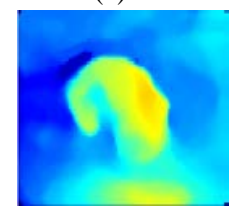

(f)
Figure 5. DTM generation procedure of the forest example (a) panchromatic images ; (b) original DSM; (c) DTM1; (d) DTM2; (e)DTM3; (f) Refined DTM; (g) reference DTM.

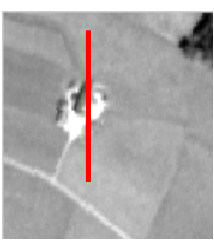

(a)

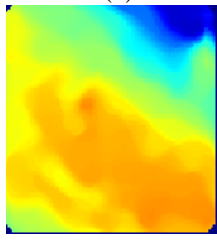

(d)

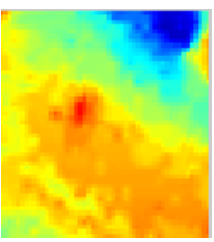

(b)

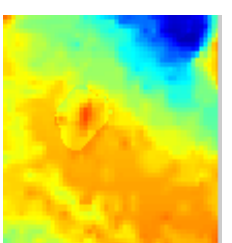

(c)

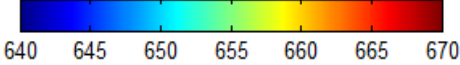

Figure 6. DTM generation procedure of building example (a) panchromatic images ; (b) original DSM; (c) DTM1; (d) refined DTM; (e) reference DTM.

To prove the advantage of our proposed method, we have compared our generated DTMs to the ones generated with tophat morphological filtering. They are both compared to the reference DTM.

Figure 7 and 8 show the profile comparison of the DSM and DTMs of the forest and buildings respectively. For better description, the profiles of the original DSMs are also shown in these two figures with black color. DTMs generated from our method and tophat have been displayed with red and blue color respectively. The reference DTM is shown with green color. As can be seen, for normal building / house, the extracted DTM matches well with the reference DTM. In the forest region, though the obtained DTM has some height difference from the reference DTM, the terrain slopes are well preserved.

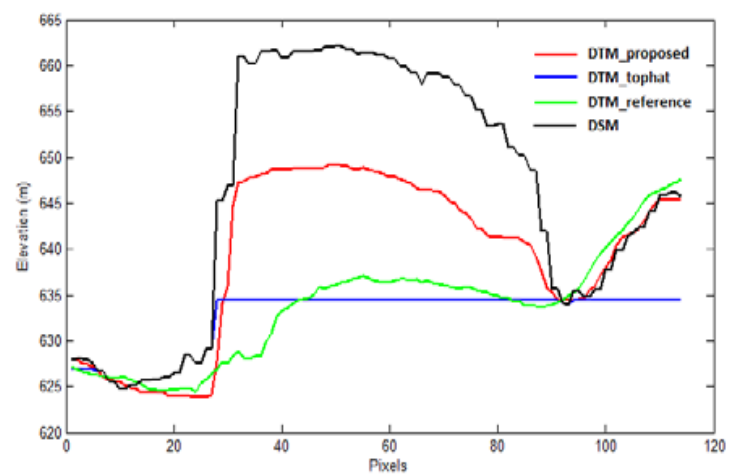

Figure 7. Profile comparison between the DTMs along the red line in Figure 5a (Black: original DSM; Red: generated DSM; Blue: tophat morphological reconstruction; Green: reference DTM). 


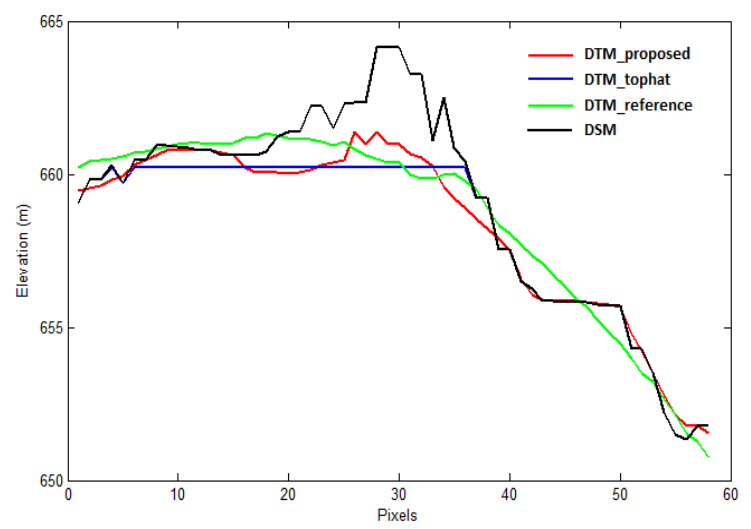

Figure 8. Profile comparison between the DTMs along the red line in Figure 6a (Black: original DSM; Red: generated DSM; Blue: tophat morphological reconstruction; Green: reference DTM).

\section{CONCLUSION}

In this paper, a new DTM generation approach is presented. In contrast to the classical approaches, the proposed method has fully used all information provided by the stereo satellite imagery. As the first contribution, an easy implementable classification refinement method is proposed. Since not enough spectral features are available for some stereo satellite images, we have used the multi-le el texture information for the classification.

Based on the classification result, a region based step-down DTM generation procedure is proposed. In our approach, we suppose that trees in the same region exhibit the same or similar height. The generated DSM can preserve the original terrain information. However, in some cases, the trees in the middle of the region can be higher than other trees. Therefore, the generated DTM is much higher than the reference DTM. This problem will be solved in the future work.

\section{Reference:}

Arefi, H., d'Angelo, P., Mayer, H., and Reinartz, P., 2011. Iterative approach for efficient digital terrain model production from CARTOSAT-1 stereo images. Journal of Applied Remote Sensing, vol. 5, no.1, pp. 1-19.

Baltsavias, E. P., 1999. A comparison between photogrammetry and laser scanning, ISPRS Journal of photogrammetry and Remote Sensing, vol. 54, no. 2, pp. 83-94.

Breiman, L., 2001. Random Forest. Machine Learning, vol. 45, no. 1 , pp. 5-32.

d'Angelo, P., 2010. Image matching and outlier removal for large scale DSM generation. Convergence in Geomatics. CGC \& ISPRS. ISPRS Symposium Commission I, 15.-18. June 2010, Calgary, Canada.

Kraus, K. and Pfeifer, N., 2001. Advanced DTM generation from LIDAR data. International Archives of Photogrammetry Remote Sensing and Spatial Information Sciences, vol. 34, no. 3/W4, pp. 23-30.

Krauss, T., Arefi, H. and Reinartz, P., 2011. Evaluation of selected methods for extracting digital terrain models from satellite born digital surface models in urban areas. International Conference on Sensors and Models in Photogrammetry and Remote Sensing (SMPR 2011), pp.1 7.

Pfeifer, N., Stadler, P., Briese, C., 2001. Derivation of digital terrain models in the SCOP++ environment. Proceedings of OEEPE Workshop on Airborne Laserscanning and Interferometric SAR for Detailed Digital Elevation Models, Stockholm, Sweden (2001) (13p.)

Razak, K. A., Santangelo, M., Westen, C. J. V., Straatsma, M. W. and de Jong, S. M., 2013. Generating an optimal DTM from airborne laser scanning data for landslide mapping in a tropical forest environment. Geomorphology, vol. 190, pp. 112-125.

Straub, C., Tian, J., Seitz, R., and Reinartz, P., 2013. Assessment of Cartosat-1 and WorldView-2 stereo imagery in combination with a LiDAR DTM for timber volume estimation in a highly structured forest in Germany. Forestry, vol. 86, pp. 463-473.

Tian, J., Reinartz, P., d'Angelo, P. and Ehlers, M., 2013. Region-based automatic building and forest change detection on Cartosat-1 stereo imagery. ISPRS Journal of Photogrammetry and Remote Sensing, vol. 79, pp. 229239.

Zhang, L., 2005. Automatic Digital Surface Model (DSM) Generation from Linear Array Images. PhD Thesis, Swiss Federal Institute of Technology Zürich, Switzerland. 